Interesting Images

\title{
Coronary Artery Disease and Gallbladder Inflammatory Pseudopolyps
}

\author{
Margherita Fosio $^{1}$, Giulia Cherobin ${ }^{1}$, Roberto Stramare ${ }^{1}$, Matteo Fassan ${ }^{2,3}$ (D) and Chiara Giraudo ${ }^{1, *(D)}$ \\ 1 UOSD Imaging Avanzato Clinico e Translazionale, Department of Medicine, University of Padova, \\ 35127 Padova, Italy; margherita.fosio@gmail.com (M.F.); giuliachero@gmail.com (G.C.); \\ roberto.stramare@unipd.it (R.S.) \\ 2 Surgical Pathology, Department of Medicine, University of Padova, 35121 Padova, Italy; \\ matteo.fassan@unipd.it \\ 3 Veneto Institute of Oncology, IOV-IRCCS, 35128 Padova, Italy \\ * Correspondence: chiara.giraudo@unipd.it
}

check for updates

Citation: Fosio, M.; Cherobin, G.; Stramare, R.; Fassan, M.; Giraudo, C. Coronary Artery Disease and Gallbladder Inflammatory Pseudopolyps. Diagnostics 2022, 12, 155. https://doi.org/10.3390/ diagnostics12010155

Academic Editor: Kazushi Numata

Received: 17 December 2021

Accepted: 8 January 2022

Published: 10 January 2022

Publisher's Note: MDPI stays neutral with regard to jurisdictional claims in published maps and institutional affiliations.

Copyright: (C) 2022 by the authors. Licensee MDPI, Basel, Switzerland. This article is an open access article distributed under the terms and conditions of the Creative Commons Attribution (CC BY) license (https:// creativecommons.org/licenses/by/ $4.0 /)$.

\begin{abstract}
Axial MR image demonstrating multiple small gallbladder polypoid lesions characterized by contrast enhancement in a 78-year-old male hospitalized for acute chest pain due to coronary artery disease who showed fever and emesis during hospitalization and had signs of acute acalculous cholecystitis at computed tomography. Given the overall clinical conditions and the MR features, the inflammatory origin of the polyps was considered. The patient underwent cholecystectomy and the histological diagnosis of gallbladder inflammatory pseudopolyps was confirmed. This rare entity represents $5-10 \%$ of all gallbladder polyps, and their differentiation from benign and malignant tumors might be challenging especially in acalculous patients, thus surgery is often performed.
\end{abstract}

Keywords: gallbladder inflammatory polyps; MR; pseudotumor

A 78-year-old man was admitted to the emergency room of our tertiary center for acute chest pain due to coronary artery disease and underwent coronary artery bypass. During the hospitalization he had fever and emesis. Thus, he underwent contrast enhanced computed tomography demonstrating acute acalculous cholecystitis with localized gallbladder perforation (Figure 1a). Ultrasound-guided percutaneous transhepatic cholecystostomy was performed and antibiotic therapy administered. Ninety days later, a Magnetic Resonance (MR) scan showed multiple small gallbladder polyps characterized by contrast enhancement without any evidence of gallbladder stones (Figure 1b). The radiological findings were compatible with an inflammatory etiology of the polypoid lesions although, especially because of the lack of gallbladder stones and previous MR scans, neoplastic polyps could not be completely excluded. The patient underwent cholecystectomy and the histological diagnosis of gallbladder inflammatory pseudopolyps (GIP) was confirmed (Figure 2a,b).

Acute acalculous cholecystitis, even complicated by perforation, often occurs in postsurgical adults while in children it is often caused by infectious disease or immune-mediated disorders. Moreover, it can be associated with cardiovascular diseases, in particular with coronary artery disease and in children with Kawasaki Disease [1-4]. The etiology of acalculous cholecystitis is often unknown, even though it has been correlated with biliary hypokinesia and local ischemia [5-8].

The radiological literature regarding rare pseudotumors like GIP, which represents $5-10 \%$ of all gallbladder polyps, is scarce, and, even if they are usually multiple and small ( $<10 \mathrm{~mm})$, a differentiation from benign and malignant tumors might be challenging. Mucosal irritation, granulation, and fibrous tissue, usually due to gallstones and/or chronic inflammation may cause GIPs [9-11].

The rarity of our case in which the occurrence of GIP is probably related to acalculous cholecystitis subsequent to acute coronary artery disease, is highlighted by the results of 
the brief literature search, without any restrictions on language and publication date, we conducted on Pubmed on the 4 January 2022. Indeed, using the keywords "(gallbladder inflammatory pseudopolyps) AND (coronary artery disease)" no records could be identified while applying the keywords "(acute acalculous cholecystitis) AND (coronary artery disease)" and "(acalculous cholecystitis) AND (inflammatory polyps)", only 16 and one records were identified, respectively [12-28]. Three of the 16 records were excluded because they were not fully matching [25-27] and the single record deriving from the second search is not reported in Table 1 since it is a narrative review [28]. The records of the search are summarized in Table 1.
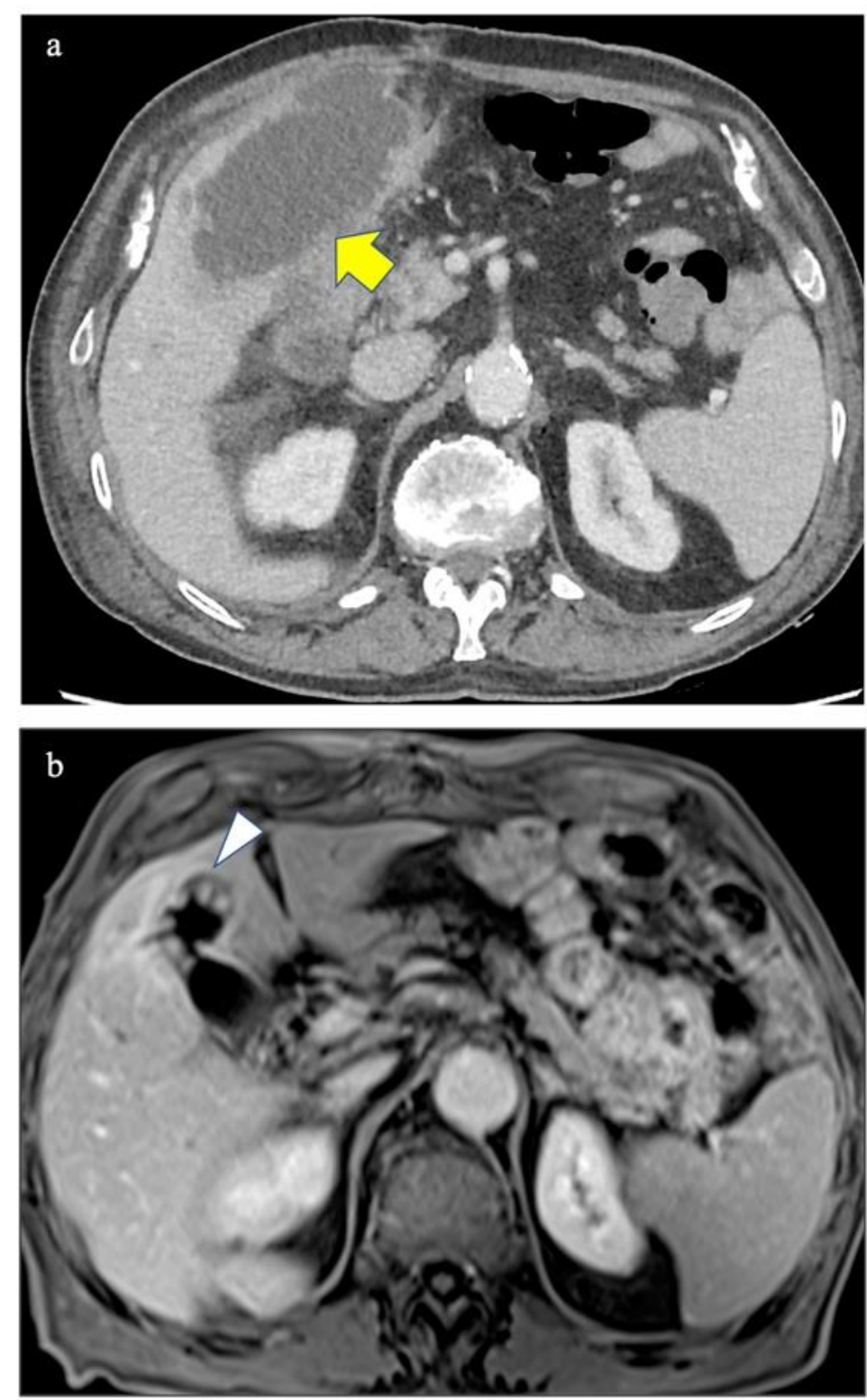

Figure 1. Axial contrast-enhanced Computed Tomography image of the upper abdomen welldemonstrating the acute cholecystitis with localized perforation (yellow arrow in (a)) and fat-saturated Volume Interpolated Breath-Hold Examination (VIBE) Magnetic Resonance image after contrast injection performed 90 days later showing multiple small pseudopolyps in the fundus of the gallbladder (white arrow in (b)). 

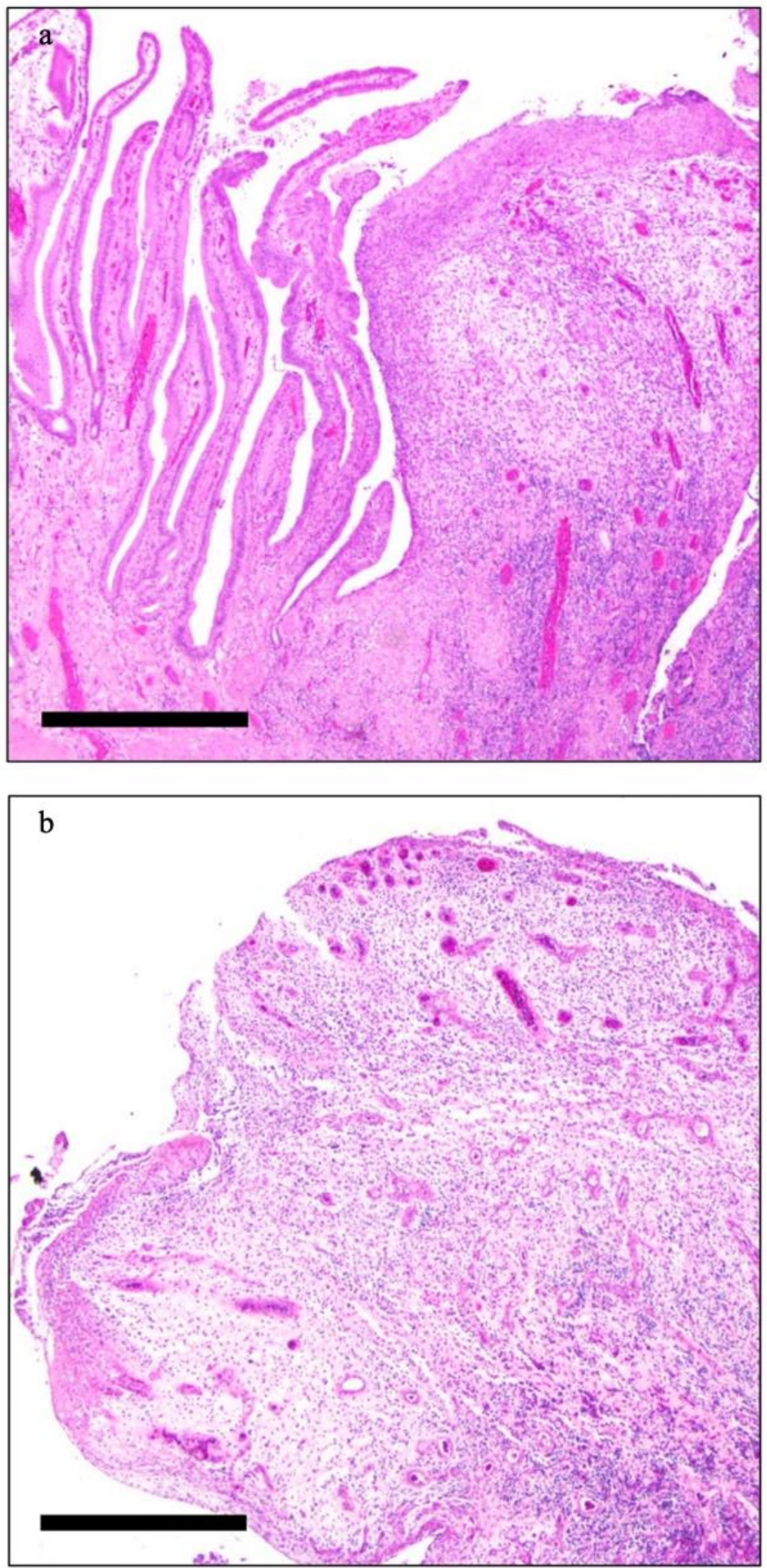

Figure 2. Histological image demonstrating papillary hyperplasia and an inflammatory pseudopolyp (a) and particular representative picture of one of the described inflammatory pseudopolyps observed in the gallbladder showing an ulcerated sessile mucosal projection characterized by edematous vascularized stroma with mixed type inflammatory infiltration (b) (scale bar $500 \mathrm{um}$ ). 
Table 1. Summary of the records collected by a brief Pubmed literature search using the keywords "(acute acalculous cholecystitis) AND (coronary artery disease)".

\begin{tabular}{|c|c|c|c|c|c|}
\hline $\begin{array}{l}\text { Publication } \\
\text { Year }\end{array}$ & First Author & Title & Study Type & Number of Cases & $\begin{array}{c}\text { Main Findings Related } \\
\text { to AAC and Coronary } \\
\text { Artery Disease }\end{array}$ \\
\hline 1986 & $\begin{array}{l}\text { Welling, R.E., } \\
\text { et al. [12] }\end{array}$ & $\begin{array}{l}\text { Gastrointestinal } \\
\text { complications after } \\
\text { cardiac surgery }\end{array}$ & Original Article & $\begin{array}{l}18 \text { patients out of } \\
1596 \text { who } \\
\text { underwent } \\
\text { coronary artery } \\
\text { bypass or valve } \\
\text { replacement. had } \\
\text { gastrointestinal } \\
\text { complications }\end{array}$ & $\begin{array}{l}\text { One patient underwent } \\
\text { cholecystectomy for AAC }\end{array}$ \\
\hline
\end{tabular}

\begin{tabular}{|c|c|c|c|c|c|}
\hline 1988 & $\begin{array}{l}\text { Varma, D.G., } \\
\text { et al. [13] }\end{array}$ & $\begin{array}{l}\text { Computed } \\
\text { tomography of } \\
\text { gangrenous acute } \\
\text { postoperative } \\
\text { acalculous } \\
\text { cholecystitis }\end{array}$ & Case report & 1 & $\begin{array}{c}\text { Gangrenous AAC after } \\
\text { two-vessel coronary artery } \\
\text { bypass surgery }\end{array}$ \\
\hline 1989 & $\begin{array}{l}\text { Berger, H. } \\
\text { et al. [14] }\end{array}$ & $\begin{array}{l}\text { Percutaneous } \\
\text { cholecystostomy in } \\
\text { acute acalculous } \\
\text { cholecystitis }\end{array}$ & Original Article & 8 & $\begin{array}{l}\text { PC was successful in all } \\
\text { patients with AAC. One } \\
\text { patient had in anamnesis a } \\
\text { coronary bypass operation }\end{array}$ \\
\hline 1993 & $\begin{array}{l}\text { Teranishi, K., } \\
\text { et al. }{ }^{\#}[15]\end{array}$ & $\begin{array}{l}\text { A case of acute } \\
\text { hemorrhagic } \\
\text { gangrenous acalculous } \\
\text { cholecystitis with bile } \\
\text { peritonitis during } \\
\text { anti-coagulant therapy } \\
\text { after coronary-artery } \\
\text { bypass grafting }\end{array}$ & Case report & 1 & $\begin{array}{l}\text { A case of acute } \\
\text { hemorrhagic, gangrenous } \\
\text { acalculous cholecystitis } \\
\text { after coronary-artery } \\
\text { bypass grafting. } \\
\text { Post-operative stasis of } \\
\text { bile, swelling of the } \\
\text { gallbladder, hypotension } \\
\text { during cardiopulmonary } \\
\text { bypass, and } \\
\text { post-operative } \\
\text { anti-coagulant therapy } \\
\text { administered after open } \\
\text { heart surgery have been } \\
\text { proposed as etiological } \\
\text { factors }\end{array}$ \\
\hline 1997 & $\begin{array}{l}\text { Saito, A., } \\
\text { et al. [16] }\end{array}$ & $\begin{array}{c}\text { Acute acalculous } \\
\text { cholecystitis after } \\
\text { cardiovascular surgery }\end{array}$ & Original Article & 6 & $\begin{array}{c}\text { Examination of six cases } \\
\text { of AAC after } \\
\text { cardiovascular surgery } \\
\text { and AAC. The authors } \\
\text { suggest that post-surgical } \\
\text { hypoperfusion of the } \\
\text { gallbladder due to various } \\
\text { factors may be the cause }\end{array}$ \\
\hline 1999 & $\begin{array}{l}\text { Fujiii, H., } \\
\text { et al. [17] }\end{array}$ & $\begin{array}{l}\text { Acute acalculous } \\
\text { cholecystitis } \\
\text { complicated by } \\
\text { penetration into the } \\
\text { liver after coronary } \\
\text { artery bypass grafting }\end{array}$ & Case report & 1 & $\begin{array}{l}\text { AAC with penetration } \\
\text { into the liver in a } \\
\text { 71-year-old woman } \\
21 \text { days after coronary } \\
\text { artery bypass grafting. At } \\
\text { histology, partial } \\
\text { obstruction of the cystic } \\
\text { artery due to } \\
\text { atherosclerosis was found }\end{array}$ \\
\hline
\end{tabular}


Table 1. Cont.

\begin{tabular}{|c|c|c|c|c|c|}
\hline $\begin{array}{l}\text { Publication } \\
\text { Year }\end{array}$ & First Author & Title & Study Type & Number of Cases & $\begin{array}{c}\text { Main Findings Related } \\
\text { to AAC and Coronary } \\
\text { Artery Disease }\end{array}$ \\
\hline 2003 & $\begin{array}{l}\text { Funabiki, K., } \\
\text { et al. [18] }\end{array}$ & $\begin{array}{l}\text { Cholesterol crystal } \\
\text { embolization (CCE) } \\
\text { after cardiac } \\
\text { catheterization: a case } \\
\text { report and a review of } \\
36 \text { cases in the } \\
\text { Japanese literature }\end{array}$ & Case Report & 1 & $\begin{array}{c}\text { A 67-year old man } \\
\text { developed AAC } 12 \text { days } \\
\text { after coronary } \\
\text { angiography which } \\
\text { followed a previous } \\
\text { coronary artery bypass } \\
\text { grafting }\end{array}$ \\
\hline 2012 & $\begin{array}{l}\text { Chen, C.J., } \\
\text { et al. [19] }\end{array}$ & $\begin{array}{l}\text { Sonographic } \\
\text { gallbladder } \\
\text { abnormality is } \\
\text { associated with } \\
\text { intravenous } \\
\text { immunoglobulin } \\
\text { resistance in Kawasaki } \\
\text { disease }\end{array}$ & Original Article & $\begin{array}{l}93 \text { children with } \\
\text { Kawasaki Disease }\end{array}$ & $\begin{array}{l}\text { Five children with KD out } \\
\text { of } 11 \text { with pathologic } \\
\text { findings at abdominal } \\
\text { ultrasound had AAC. } \\
\text { Overall pathologic } \\
\text { findings at US in children } \\
\text { with KD seem to be } \\
\text { associated with high } \\
\text { levels of C-reactive } \\
\text { protein, Glutamic-Pyruvic } \\
\text { Transaminase, neutrophils } \\
\text { and intravenous } \\
\text { immunoglobulin } \\
\text { resistance }\end{array}$ \\
\hline 2012 & $\begin{array}{l}\text { Van Stejin, } \\
\text { J.H.M., et al. } \\
\quad{ }^{*}[20]\end{array}$ & $\begin{array}{c}\text { Acute acalculous } \\
\text { cholecystitis: not only } \\
\text { in the intensive care } \\
\text { department }\end{array}$ & Case Reports & 2 & $\begin{array}{l}\text { Two patients with AAC } \\
\text { are reported; one of them } \\
\text { admitted to the coronary } \\
\text { unit because of } \\
\text { atherosclerotic vascular } \\
\text { disease then died of sepsis }\end{array}$ \\
\hline 2014 & $\begin{array}{l}\text { Yi, D., et al. } \\
\text { [21] }\end{array}$ & $\begin{array}{l}\text { Hepatobiliary risk } \\
\text { factors for clinical } \\
\text { outcome of Kawasaki } \\
\text { disease in children }\end{array}$ & Original Article & $\begin{array}{l}24 \text { out of } 67 \\
\text { children with KD } \\
\text { had AAC }\end{array}$ & $\begin{array}{c}\text { Coronary artery } \\
\text { abnormalities were more } \\
\text { frequent in patients with } \\
\text { AAC }\end{array}$ \\
\hline 2019 & $\begin{array}{l}\text { Kang, W.D., } \\
\text { et al. [22] }\end{array}$ & $\begin{array}{l}\text { Clinical aspects of } \\
\text { splenomegaly as a } \\
\text { possible predictive } \\
\text { factor of coronary } \\
\text { artery changes in } \\
\text { Kawasaki disease }\end{array}$ & Original Article & $\begin{array}{c}77 \text { out of } 396 \\
\text { examined patients } \\
\text { underwent } \\
\text { abdominal } \\
\text { ultrasound }\end{array}$ & $\begin{array}{l}\text { There were no cases of } \\
\text { AAC at ultrasound among } \\
\text { all investigated patients }\end{array}$ \\
\hline 2019 & $\begin{array}{l}\text { Lipe, D.N., } \\
\text { et al. [23] }\end{array}$ & $\begin{array}{c}\text { Kawasaki Disease } \\
\text { Presenting as Acute } \\
\text { Acalculous } \\
\text { Cholecystitis }\end{array}$ & Case report & 1 & $\begin{array}{c}\text { Eight-year-old boy } \\
\text { affected by KD and with } \\
\text { AAC }\end{array}$ \\
\hline 2021 & $\begin{array}{l}\text { Chen, B.Q., } \\
\text { et al. [24] }\end{array}$ & $\begin{array}{c}\text { Percutaneous } \\
\text { cholecystostomy as a } \\
\text { definitive treatment } \\
\text { for moderate and } \\
\text { severe acute } \\
\text { acalculous } \\
\text { cholecystitis: a } \\
\text { retrospective } \\
\text { observational study }\end{array}$ & Original Article & 44 & $\begin{array}{l}\text { In patients with moderate } \\
\text { to severe AAC who } \\
\text { underwent PC, coronary } \\
\text { heart disease or } \\
\text { congestive heart failure } \\
\text { are independent risk } \\
\text { factors for relapse }\end{array}$ \\
\hline
\end{tabular}

$\mathrm{PC}=$ percutaneous cholecystostomy; $\mathrm{AAC}=$ acute acalculous cholecystitis; $\mathrm{KD}=$ Kawasaki Disease; ${ }^{\#}$ information extracted from the abstract only since the full-text was not in English. 
Thus, our case demonstrates that, although rarely, GIP may occur in patients with acalculous cholecystitis even associated with coronary artery disease and can be easily diagnosed at MR. Given the clinical course of our patient, the etiology and the benign nature of the pseudopolyps could have been assumed. Nevertheless, since the malignant behavior of gallbladder lesions is not easily excluded at imaging, especially in acalculous and symptomatic patients with an unknown/partially known clinical history, surgery still plays a dominant role and histology remains the gold standard for a precise characterization.

Author Contributions: M.F. (Margherita Fosio) and C.G.: Conceptualization. G.C. and R.S.: collected clinical information; M.F. (Margherita Fosio): writing-original draft preparation; C.G., G.C., R.S. and M.F. (Matteo Fassan): writing-review and editing; G.C. and M.F. (Matteo Fassan): visualization; C.G.: supervision. All authors have read and agreed to the published version of the manuscript.

Funding: This research received no external funding.

Institutional Review Board Statement: Not applicable.

Informed Consent Statement: The patient provided informed consent for the description and publication of the case.

Conflicts of Interest: The authors declare no conflict of interest.

\section{References}

1. Tana, M.; Tana, C.; Cocco, G.; Iannetti, G.; Romano, M.; Schiavone, C. Acute acalculous cholecystitis and cardiovascular disease: A land of confusion. J. Ultrasound 2015, 18, 317-320. [CrossRef] [PubMed]

2. Cher, D.J. Myocardial Infarction and Acute Cholecystitis: An Application of Sequence Symmetry Analysis. Epidemiology 2000, 11, 446-449. [CrossRef] [PubMed]

3. Rezkallah, K.N.; Barakat, K.; Farrah, A.; Rao, S.; Sharma, M.; Chalise, S.; Zdunek, T. Acute Acalculous Cholecystitis due to primary acute Epstein-Barr virus infection treated with laparoscopic cholecystectomy: A case report. Ann. Med. Surg. 2018, 35, 189-191. [CrossRef] [PubMed]

4. Huffmann, J.L.; Schenker, S. Acute acalculous cholecystitis: A review. Clin. Gastroenterol. Hepatol. 2010, 8, 15-22. [CrossRef]

5. Chen, P.F.; Nimeri, A.; Pham, Q.H.; Yuh, J.N.; Gusz, J.R.; Chung, R.S. The clinical diagnosis of chronic acalculous cholecystitis. Surgery 2001, 130, 578-581. [CrossRef]

6. Wiles, R.; Thoeni, R.F.; Barbu, S.T.; Vashist, Y.K.; Rafaelsen, S.R.; Dewhurst, C.; Arvanitakis, M.; Lahaye, M.; Soltes, M.; Perinel, J.; et al. Management and follow-up of gallbladder polyps. Eur. Radiol. 2017, 27, 3856-3866. [CrossRef]

7. Maheshwarappa, R.P.; Menda, Y.; Graham, M.M.; Boukhar, S.A.; Zamba, G.K.D.; Samuel, I. Association of gallbladder hyperkinesia with acalculous chronic cholecystitis: A case-control study. Surgery 2020, 168, 800-808. [CrossRef]

8. Poddighe, D.; Sazonov, V. Acute acalculous cholecystitis in children. World J. Gastroenterol. 2018, 24, 4870-4879. [CrossRef]

9. Mellnick, V.M.; Menias, C.O.; Sandrasegaran, K.; Hara, A.K.; Kielar, A.Z.; Brunt, E.M.; Doyle, M.B.M.; Dahiya, N.; Elsayes, K.M. Polypoid lesions of the gallbladder: Disease spectrum with pathologic correlation. Radiographics 2015, 35, 387-399. [CrossRef]

10. Kwon, W.; Jang, J.Y.; Lee, S.E.; Hwang, D.W.; Kim, S. Clinicopathologic features of polypoid lesions of the gallbladder and risk factors of gallbladder cancer. J. Korean Med. Sci. 2009, 24, 481-487. [CrossRef]

11. Lee, K.F.; Wong, J.; Li, J.C.; Lai, P.B.S. Polypoid lesions of the gallbladder. Am. J. Surg. 2004, 188, 186-190. [CrossRef]

12. Welling, R.E.; Rath, R.; Albers, J.E.; Glaser, R.S. Gastrointestinal complications after cardiac surgery. Arch. Surg. 1986, 121, 1178-1180. [CrossRef]

13. Varma, D.G.; Faust, J.M. Computed tomography of gangrenous acute postoperative acalculous cholecystitis. J. Comput. Tomogr. 1988, 12, 29-31. [CrossRef]

14. Berger, H.; Pratschke, E.; Arbogast, H.; Stäbler, A. Percutaneous cholecystostomy in acute acalculous cholecystitis. Hepatogastroenterology 1989, 36, 346-348. [PubMed]

15. Teranishi, K.; Murase, M.; Maeda, M.; Murakami, F. A case of acute hemorrhagic gangrenous acalculous cholecystitis with bile peritonitis during anti-coagulant therapy after coronary-artery bypass grafting. Nihon Kyobu Geka Gakkai 1993, $41,83-87$.

16. Saito, A.; Shirai, Y.; Ohzeki, H.; Hayashi, J.; Eguchi, S. Acute acalculous cholecystitis after cardiovascular surgery. Surg. Today 1997, 27, 907-909. [CrossRef] [PubMed]

17. Fujiii, H.; Kubo, S.; Tokuhara, T.; Suehiro, S.; Yamamoto, T.; Kinoshita, H. Acute acalculous cholecystitis complicated by penetration into the liver after coronary artery bypass grafting. Jpn. J. Thorac. Cardiovasc. Surg. 1999, 47, 518-521. [CrossRef] [PubMed]

18. Funabiki, K.; Masuoka, H.; Shimizu, H.; Emi, Y.; Mori, T.; Ito, M.; Nakano, T. Cholesterol crystal embolization (CCE) after cardiac catheterization: A case report and a review of 36 cases in the Japanese literature. Jpn. Heart J. 2003, 44, 767-774. [CrossRef] 
19. Chen, C.J.; Huang, F.C.; Tiao, M.M.; Huang, Y.H.; Lin, L.Y.; Yu, H.R.; Yang, K.D.; Huang, Y.C.; Chen, C.C.; Chang, W.C.; et al. Sonographic gallbladder ab-normality is associated with intravenous immunoglobulin resistance in Kawasaki disease. Sci. World J. 2012, 2012, 485758. [CrossRef]

20. Van Steijn, J.H.M.; Roeloffzen, W.W.H.; Appeltans, B.M.G.; Jager, P.L.; Gans, R.O.B.; Bijl, M. Acute acalculous cholecystitis: Not only in the intensive care department. Ned. Tijdschr. Voor Geneeskd. 2002, 146, 1305-1308.

21. Yi, D.; Kim, J.Y.; Choi, E.Y.; Choi, J.Y.; Yang, H.R. Hepatobiliary risk factors for clinical outcome of Kawasaki disease in children. BMC Pediatr. 2014, 14, 51. [CrossRef]

22. Kang, D.W.; Kim, S.H. Clinical aspects of splenomegaly as a possible predictive factor of coronary artery changes in Kawasaki disease. Cardiol. Young 2019, 29, 297-302. [CrossRef] [PubMed]

23. Lipe, D.N.; Bridges, L.C. Kawasaki Disease Presenting as Acute Acalculous Cholecystitis. Clin. Pract. Cases Emerg. Med. 2019, 3, 383-386. [CrossRef] [PubMed]

24. Chen, B.Q.; Chen, G.D.; Xie, F.; Li, X.; Mao, L.; Jia, B. Percutaneous cholecystostomy as a definitive treatment for moderate and severe acute acalculous cholecystitis: A retrospective observational study. BMC Surg. 2021, 21, 439. [CrossRef]

25. Shapiro, M.J.; Luchtefeld, W.B.; Kurzweil, S.; Kaminski, D.L.; Durham, R.M.; Mazuski, J.E. Acute acalculous cholecystitis in the critically ill. Am. Surg. 1994, 60, 335-339.

26. Tsuboi, I.; Hayashi, M.; Miyauchi, Y.; Iwasaki, Y.K.; Yodogawa, K.; Hayashi, H.; Uetake, S.; Takahashi, K.; Shimizu, W. Anatomical factors associated with periesophageal vagus nerve injury after catheter ablation of atrial fibrillation. J. Nippon. Med. Sch. 2014, 81, 248-257. [CrossRef] [PubMed]

27. Udekwu, P.O.; Sullivan, W.G. Contemporary experience with cholecystectomy: Establishing 'benchmarks' two decades after the introduction of laparoscopic cholecystectomy. Am. Surg. 2013, 79, 1253-1257. [CrossRef]

28. Owen, C.; Bilhartz, L.E. Gallbladder polyps, cholesterolosis, adenomyomatosis, and acute acalculous cholecystitis. Semin. Gastrointest. Dis. 2003, 14, 178-188. 